\title{
Overexpression of Tiam1 is associated with malignant phenotypes of nasopharyngeal carcinoma
}

\author{
YI DING $^{1 *}$, BIN CHEN $^{2 *}$, JING HUANG ${ }^{1,3}$, WENLI ZHANG ${ }^{4-6}$, HONGJUN YANG $^{4-6}$, \\ YONGJIAN DENG ${ }^{4-6}$, JIE LIN ${ }^{4-6}$, SHUANG WANG ${ }^{4-6}$ and XIANGMEI ZHANG ${ }^{4,5,7}$ \\ ${ }^{1}$ Department of Radiation Oncology, Nanfang Hospital, Southern Medical University, Guangzhou, Guangdong 510515; \\ ${ }^{2}$ Department of Academic Research and Medical Training, General Hospital of Guangzhou Military Command of PLA, \\ Guangzhou, Guangdong 510515; ${ }^{3}$ Cancer Center of the Affiliated Hospital, Guangdong Medical College, Zhanjiang, \\ Guangdong 524001; ${ }^{4}$ Department of Pathology, School of Basic Medical Sciences, Southern Medical University, \\ Guangzhou, Guangdong 510515; ${ }^{5}$ Department of Pathology, Nanfang Hospital, Southern Medical University, \\ Guangzhou, Guangdong 510515; ${ }^{6}$ Guangdong Provincial Key Laboratory of Molecular Tumor Pathology, \\ Guangzhou, Guangdong 510515; ${ }^{7}$ Department of Pathology, Shenzhen Third People's Hospital, \\ Shenzhen, Guangdong 518020, P.R. China
}

Received February 21, 2014; Accepted May 20, 2014

DOI: $10.3892 /$ or.2014.3241

\begin{abstract}
The aim of the present study was to analyze the roles of T lymphoma invasion and metastasis 1 (Tiam1) in nasopharyngeal carcinoma (NPC) progression and its correlation with clinicopathological features, including the survival of patients with NPC. Tiam1 protein expression in NPC tissues was examined using immunohistochemistry. Reverse transcriptionpolymerase chain reaction (RT-PCR) and immunofluorescence staining were performed to detect the expression of Tiam1 in 6 NPC cell lines. Stable Tiam1-overexpressing NPC cells using a transfection technique and Tiam1-silencing NPC cells using short hairpin RNA were constructed. Subsequently, MTT assay, plate and soft agar colony formation assays, cell adhesion, migration, invasion assays and experimental animal models were carried out to detect the biological functions of Tiam1 in vitro and in vivo. Immunohistochemical analysis revealed that Tiam1 had high expression in 96 of 140 (68.6\%) paraffin-embedded archival NPC biopsies. Tiam1 overexpression was significantly associated with $\mathrm{N}$ classification $(\mathrm{P}=0.004)$, distant metastasis $(\mathrm{P}=0.042)$ and clinical stage $(\mathrm{P}=0.042)$. Patients with higher levels of Tiam1 expression had poorer overall survival $(\mathrm{P}=0.002)$. Multivariate analysis revealed that Tiam1 expression is an independent prognostic
\end{abstract}

Correspondence to: Professor Shuang Wang or Professor Xiangmei Zhang, Department of Pathology, Nanfang Hospital, Southern Medical University, Guangzhou, Guangdong 510515, P.R. China

E-mail: wztg8579@163.com

E-mail: zxmtg2014@126.com

*Contributed equally

Key words: Tiam1, NPC, prognosis, tumor malignant phenotype indicator for the overall survival of NPC patients. Using the approaches of exogenous overexpression and the knockdown of Tiam1 expression, respectively, it was confirmed that Tiam1 promoted cell proliferation, adhesion, invasion and migration in vitro and in vivo. These data support the notion that Tiam1 plays an important role in the progression of NPC, and the overexpression of Tiam1 is associated with malignant phenotypes of NPC.

\section{Introduction}

Nasopharyngeal carcinoma (NPC), a malignant tumor arising from the epithelium of the nasopharynx, has a unique geographical and ethnic distribution. As one of the most common cancers found among individuals of Chinese or Asian ancestry, it poses one of the most serious health problems in southern China where an annual incidence of more than 20 cases per 100,000 is reported (1). A number of studies indicate that the etiology of NPC is associated with multiple factors, including genetics, Epstein-Barr virus and environment (2). Patients with NPC tend to present at a more advanced stage of disease as the primary anatomical site of tumor growth is located in a silent area, and NPC exhibits higher metastatic potential when compared with other head and neck squamous cell carcinomas $(3,4)$. Despite being potentially curable at an early stage, more than $50 \%$ of NPC patients present with advanced locoregional disease, which results in a poor patient prognosis (5). Therefore, the screening and early detection in a population at risk have been proposed to decrease both the morbidity and the mortality associated with NPC.

T lymphoma invasion and metastasis 1 (Tiam1), a specific guanine nucleotide exchange factor (GEF) for Rac1 and an important member of the Rho GTPase family, was first identified as a gene which might induce invasion and metastasis by proviral tagging, in combination with in vitro selection for invasiveness in T lymphoma cells (6). The role of Tiam1 
in cellular migration, invasion and metastasis may not be limited to $\mathrm{T}$ lymphoma. It has been reported to be significant in promoting tumor progression in a variety of cancers, such as breast, colorectal, lung, liver cancer and Ras-induced skin tumors (7-11). Recent study indicates that Tiam1 has different effects on various types of cancers. Uhlenbrock et al (12) found that Tiam1 inhibited the migration and invasion of metastatic melanoma via a novel adhesive mechanism. However, little is known concerning the potential prognostic value and molecular mechanisms of Tiam1 in NPC.

In the present study, we aimed to investigate the endogenous expression of Tiam1 in primary NPC tissues and cell lines and to identify the relationship between Tiam1 expression and clinicopathological features, including the survival of patients with NPC. Furthermore, we observed the effects of intentionally regulated changes in Tiam1 expression levels on the in vivo and in vitro functions of NPC cells. The present study aimed to evaluate the prognostic value of Tiam1, in regards to patient survival in NPC and to explore the essential role of Tiam1 in tumor cell growth, migration, invasion and metastasis in NPC.

\section{Materials and methods}

Patients and tissue specimens. A total of 140 previously untreated patients with NPC, who were histologically and clinically diagnosed at the Nanfang Hospital, Southern Medical University, China, between 1998 and 2000, were enrolled in the present study. For the use of these clinical materials for research purposes, prior patient consent and approval from the Ethics Committee of the Southern Medical University were obtained. The stage of cancer was defined according to the 1992 Fuzhou NPC staging system of China. Clinical information concerning the samples is described in detail in Table I. The patients included 104 males and 36 females, ranging in age from 20 to 74 years (mean, 48.3 years). The median follow-up time for overall survival was 41.2 months for patients still alive at the time of analysis, and ranged from 1.83 to 80.6 months. In addition, 30 patients with chronic nasopharyngitis were used as the control.

Cell lines and animals. The human NPC cell lines, CNE1, CNE2, HONE1 and C666-1, were obtained from the Cancer Institute, Southern Medical University (Guangzhou, China). 5-8F (high tumorigenic and metastatic ability) and 6-10B (tumorigenic, but lacking metastatic ability) cells from colony lines of the NPC SUNE1 cell line (13) were provided by the Cancer Center of Sun Yet-Sen University (Guangzhou, China). These cell lines were maintained in RPMI-1640 medium (Gibco, Grand Island, NY, USA) supplemented with $10 \%$ heat-inactivated fetal bovine serum (FBS) and $100 \mathrm{U} / \mathrm{ml}$ of penicillin/streptomycin at $37^{\circ} \mathrm{C}$ in a humidified atmosphere of $5 \% \mathrm{CO}_{2}$ in air. The $293 \mathrm{~T}$ cells were cultured in Dulbecco's modified Eagle's medium (Gibco). Female nude mice (6- to 8-weeks old and 16-21 g in weight) were purchased from the Central Laboratory of Animal Science, Southern Medical University. All animals were housed under standard pathogenfree conditions. All animal experiments were performed with approval from the Institutional Animal Ethics Committees of the Southern Medical University.
Table I. Correlation between the clinicopathological features and expression of Tiam1 protein.

\begin{tabular}{|c|c|c|c|c|}
\hline \multirow[b]{2}{*}{ Characteristics } & \multirow[b]{2}{*}{$\mathrm{n}$} & \multicolumn{2}{|c|}{ Tiam1 (\%) } & \multirow[b]{2}{*}{ P-valu } \\
\hline & & Negative & Positive & \\
\hline \multicolumn{5}{|l|}{ Gender } \\
\hline Male & 104 & $29(27.88)$ & $75(72.12)$ & 0.125 \\
\hline Female & 36 & $15(41.67)$ & $21(58.33)$ & \\
\hline \multicolumn{5}{|l|}{ Age (years) } \\
\hline$\leq 45$ & 65 & $21(32.31)$ & $44(67.69)$ & 0.464 \\
\hline$>45$ & 75 & $20(26.67)$ & $55(73.33)$ & \\
\hline \multicolumn{5}{|c|}{ Pathological classification } \\
\hline Type II & 131 & $36(27.48)$ & $95(72.52)$ & \\
\hline Type III & 9 & $5(55.56)$ & $4(44.44)$ & 0.073 \\
\hline \multicolumn{5}{|l|}{ T classification } \\
\hline $\mathrm{T}_{1}-\mathrm{T}_{2}$ & 83 & $28(33.73)$ & $55(66.27)$ & \\
\hline $\mathrm{T}_{3}-\mathrm{T}_{4}$ & 57 & $13(22.81)$ & $44(77.19)$ & 0.163 \\
\hline \multicolumn{5}{|l|}{$\mathrm{N}$ classification } \\
\hline $\mathrm{N}_{0}$ & 91 & $34(37.36)$ & $57(62.64)$ & \\
\hline $\mathrm{N}_{1}-\mathrm{N}_{3}$ & 49 & $7(14.29)$ & $42(85.71)$ & 0.004 \\
\hline \multicolumn{5}{|c|}{ Distant metastasis } \\
\hline Yes & 15 & $1 \quad(6.67)$ & $14(93.33)$ & \\
\hline No & 125 & $40(32.00)$ & $85(68.00)$ & 0.042 \\
\hline \multicolumn{5}{|l|}{ Clinical stage } \\
\hline I-II & 60 & $23(38.33)$ & $37(61.67)$ & \\
\hline III-IV & 80 & $18(22.50)$ & $62(77.50)$ & 0.042 \\
\hline
\end{tabular}

Bold print indicates statistical significance.

Immunohistochemistry (IHC). IHC staining was performed to study altered protein expression in 140 human NPC and 30 chronic nasopharyngitis tissues using a Dako EnVision System (Dako, Carpinteria, CA, USA) following the manufacturer's recommended protocol. The procedures were carried out with standard methods. Briefly, all paraffin sections, $4 \mu \mathrm{m}$ in thickness, were baked for $1 \mathrm{~h}$ at $65^{\circ} \mathrm{C}$. Sections were deparaffinized with xylene and rehydrated with graded ethanol to distilled water. Sections were submerged in EDTA antigenic retrieval buffer ( $\mathrm{pH}$ 8.0) and subjected to microwave treatment. After being treated with $0.3 \% \mathrm{H}_{2} \mathrm{O}_{2}$ for 15 min to block the endogenous peroxidase, the sections were treated with $1 \%$ bovine serum albumin for $30 \mathrm{~min}$ to reduce non-specific binding, and then rabbit polyclonal anti-Tiam1 antibody (1:200; Santa Cruz Biotechnology) was incubated with the sections overnight at $4^{\circ} \mathrm{C}$. After washing, the sections were incubated with HRP at $4^{\circ} \mathrm{C}$ for $30 \mathrm{~min}$. For color reactions, diaminobenzidine (DAB) was used. For negative controls, the antibody was replaced by normal goat serum.

Evaluation of staining. The immunohistochemically stained tissue sections were reviewed and scored separately by two pathologists blinded to the clinical parameters. For Tiam1 assessment, the entire tissue section was scanned to assign 
the scores. The staining intensity was scored as 0 (negative), 1 (weak), 2 (medium) and 3 (strong). The extent of staining was scored as $1(0 \%), 1(1-25 \%), 2(26-50 \%), 3(51-75 \%)$ and $4(76-100 \%)$ according to the percentages of the positive staining areas in relation to the entire carcinoma-involved area or the entire section for the normal samples. The sum of the intensity and extent score was used as the final staining score (0-7) for Tiam 1 . This relatively simple, reproducible scoring method provides highly concordant results between independent evaluators and has been used in previous studies (14-16). Tumors having a final staining score of 3 or higher were considered to be positive.

RNA extraction and reverse transcription-polymerase chain reaction ( $R T-P C R)$. According to the manufacturer's instructions, total RNA from the cell lines was extracted using TRIzol reagent (Invitrogen, Carlsbad, CA, USA). DNase I-treated total RNA $(\sim 1 \mu \mathrm{g})$ from each sample was used to synthesize cDNA using SuperScript II Reverse Transcriptase (Invitrogen). The segment of the Tiam1 gene was PCR amplified from cDNA samples of NPC cell lines. The following primers were used for amplification of Tiam1 (GeneBank, NM_003253): sense primer, 5'-AAGACGTACTCAGGCCATGTCC-3' and antisense primer, 5'-GACCCAAATGTCGCAGTCAG-3'. Glyceraldehyde-3-phosphate dehydrogenase was amplified as an internal control using sense primer, 5'-AATCCCATCACC ATCTTCCA-3' and antisense primer, 5'-CCTGCTTCACCA CCTTCTTG-3'. The appropriate size of the PCR products was confirmed by agarose gel electrophoresis. 1-D Advanced software (Eastman-Kodak, Co., Stamford, CT, USA) was used to calculate the ratios of the densitometry of the Tiam 1 band to the GAPDH band, indicating the relative Tiam1 expression levels.

Immunofluorescence staining. Cells were cultured on coverslips overnight and fixed with $4 \%$ paraformaldehyde for 20 min and treated with $0.25 \%$ Triton X-100 for 10 min. After blocking in $10 \%$ normal blocking serum at room temperature for $10 \mathrm{~min}$, slides were incubated with the anti-Tiam1 antibody (Santa Cruz Biotechnologies) at $4^{\circ} \mathrm{C}$ overnight and then washed with phosphate-buffered saline (PBS) three times. Coverslips were then incubated with FITC-conjugated anti-rabbit secondary antibodies (Invitrogen) for $30 \mathrm{~min}$ at room temperature, followed by staining with 4',6-diamidino2-phenylindole (DAPI; Invitrogen).

Western blot analysis. Cells were washed twice with cold PBS and lysed on ice in RIPA buffer (1X PBS, 1\% NP-40, $0.1 \%$ SDS, $5 \mathrm{mM}$ EDTA, $0.5 \%$ sodium deoxycholate and $1 \mathrm{mM}$ sodium orthovanadate) with protease inhibitors. The protein concentration was determined by the Bradford assay (Bio-Rad Laboratories, Hercules, CA, USA). Equal amounts of proteins were separated electrophoretically on $12 \%$ SDS/polyacrylamide gels and transferred onto polyvinylidene difluoride (PVDF) membranes (Amersham Pharmacia Biotech, Piscataway, NJ, USA) and blocked in 5\% non-fat dry milk in Tris-buffered saline (TBST) (pH 7.5; $100 \mathrm{mM}$ $\mathrm{NaCl}, 50 \mathrm{mM}$ Tris and 0.1\% Tween-20). Membranes were immunoblotted with the anti-Tiam1 polyclonal antibody and the anti-GAPDH antibody (Santa Cruz Biotechnology) over- night at $4^{\circ} \mathrm{C}$, followed by their respective secondary antibodies conjugated to horseradish peroxidase (HRP). The signals were detected by enhanced chemiluminescence (ECL; Pierce, Rockford, IL, USA).

Plasmid preparation. For construction of the Tiam1knockdown vectors in mammalian cells, four human siRNAs (shRNA1, CTCAGAGCATCCACATTGA; shRNA2, GGA GATGAGATTCTTGAGA; shRNA3, CGGAAATGGTAGA GTTTCA; and shRNA4, GCAGCAAGAGTACAGAACA) that targeted Tiam1 were selected, and a human scrambled siRNA sequence (5'-TTCTCCGAACGTGTCACGT-3') possessing limited homology to human genes served as a negative control (17). The siRNAs were synthesized and subcloned into the pGC-LV vector by double digestion with AgeI and EcoRI to form VshRNA (pGC-LV recombination vector) in accordance with the manufacturer's guidelines (Shanghai GeneChem, Co., Ltd., Shanghai, China). The correct coding regions of all plasmids were confirmed by sequencing. In addition, the overexpression vector of Tiam1, Tiam1/C1199HA plasmids, was a gift from Professor J.G. Collard (6).

Stable transfections regulate the expression of the Tiaml gene. Cells were seeded onto a 6 -well plate $16 \mathrm{~h}$ before transfection. Transfection assays were performed by Lipofectamine 2000 according to the manufacturer's instructions (Invitrogen). Tiam1/C1199HA vector and pcDNA3.1 vector were transfected into CNE2 cells, respectively. Lentiviruses containing the 4 Tiam1 shRNAs or the negative control shRNA were produced by plasmid cotransfection of $293 \mathrm{~T}$ cells. The viral supernatants containing shRNA1, shRNA2, shRNA3, shRNA4 and the negative control vector were added respectively to the plates with CNE2 cells. The selections for stable integrants were carried out by culturing cells with a complete medium containing G418 (for overexpression) or blasticidin (for knockdown). Tiam1 expression in the transfectants was verified by both RT-PCR and western blot analysis.

In vitro cell growth assay. The cells were prepared at a concentration of $1 \times 10^{4}$ cells $/ \mathrm{ml}$, respectively. Aliquots $(100 \mu \mathrm{l})$ were dispensed into 96-well microtiter plates and cultured overnight. The cells were cultured for 1 to 7 days. Each subsequent day, $20 \mu \mathrm{l}$ of $5 \mathrm{mg} / \mathrm{ml}$ 3-(4,5-dimethylthiazol-2-yl)-2,5-diphenyltetrazolium bromide (MTT) (Sigma, St. Louis, MO, USA) was added to each well. After being incubated for $4 \mathrm{~h}$ at $37^{\circ} \mathrm{C}$, the supernatants were carefully removed. One hundred and fifty microliters of dimethyl sulfoxide (Sigma) was added to each well. Fifteen minutes later, the absorbance value (OD) of each well was measured with a microplate reader set at $570 \mathrm{~nm}$. All experiments were performed in triplicate.

Plate colony formation assay. Approximately $1 \times 10^{2}$ cells were added to each well ( $3 \mathrm{~cm}$ in diameter) of a 6-well culture plate, and each cell group contained three wells. After incubation at $37^{\circ} \mathrm{C}$ for 12 days, the cells were washed twice with PBS and stained with Giemsa solution. The number of colonies containing $\geq 50$ cells was counted under a microscope. Plate colony formation efficiency $=$ number of colonies/number of cells inoculated x $100 \%$. 
Soft agar assay. Cells $\left(1 \times 10^{4} / \mathrm{ml}, 20 \mu \mathrm{l}\right)$ were seeded in $0.4 \%$ agar and incubated at $37^{\circ} \mathrm{C}$ for 14 days. Colonies that grew $>50 \mu \mathrm{m}$ in diameter were counted. Colony formation efficiency $=$ number of colonies/number of cells inoculated x 100\%. Each experiment was carried out in triplicate.

In vitro invasion assay. Invasion assays were performed using a cell invasion chamber (Chemicon, Temecula, CA, USA) according to the protocol of the manufacturer. The cell invasion chamber contained a polycarbonate membrane with an $8-\mu \mathrm{m}$ pore size, over which a thin layer of ECMatrix (Chemicon) was dried. The extracellular matrix (ECM) layer occludes membrane pores, blocking non-invasive cells from migrating. In brief, warm serum-free medium was added to the top chamber of the cell invasion chamber to rehydrate the ECM layer for $2 \mathrm{~h}$ at room temperature. Tumor cells in a serum-free medium ( $300 \mu 1$ containing $1 \times 10^{5}$ cells) were added to the top chamber. The bottom chamber was prepared using $10 \%$ FBS as chemoattactant. After incubation for $24 \mathrm{~h}$, the non-invasive cells were removed with a cotton swab. The cells that had migrated through the membrane and had stuck to the lower surface of the membrane were fixed with methanol and stained with hematoxylin. For quantification, the cells were counted under a microscope in five randomly selected fields (original magnification, $\mathrm{x} 200$ ).

Cell adhesion assay. To investigate the ability of cell-matrix adhesion, we analyzed the binding capacity of CNE2 cells to extracellular matrix (ECM) by seeding cells on a fibronectin substrate. Cell adhesion assay was performed as previously reported (18). Briefly, 96-well plates were firstly coated with fibronectin $\left(100 \mu \mathrm{l}, 50 \mu \mathrm{g} / \mathrm{ml}\right.$; Sigma) for $2 \mathrm{~h}$ at $37^{\circ} \mathrm{C}$. Then, cells ( $2 \times 10^{4} /$ well) were allowed to adhere to the substrate for $2 \mathrm{~h}$. After unattached cells were removed by gentle washing with PBS, the adherent cells were rinsed, fixed with $4 \%$ paraformaldehyde and quantified by $1 \%$ crystal violet staining. The OD value was measured at $570 \mathrm{~nm}$. Every group had 5 repeated wells.

Wound healing assay. Cells were cultured under standard conditions, as previously described, until $70-80 \%$ confluency. Cells were grown to $90 \%$ confluency in 6-well plates. Scratches of $1 \mathrm{~mm}$ were constructed with the tip of a micropipette, followed by addition of fresh serum-containing medium. Phase-contrast microscopic images were captured 0-30 h after the scratches were made in order to observe their closure. Migration was quantified by counting the number of cells that had moved across the starting line.

In vivo metastasis assays. The cells were first harvested by trypsinization, washed thrice with cold serum-free medium, and resuspended with serum-free medium. Six-week-old female nude mice were injected respectively with the differently treated cells, including the Tiam1-overexpressing cells, Tiam 1 shRNA cells and the corresponding control cells, via the lateral tail vein ( $\mathrm{n}=9$ per group). The mice were sacrificed 9 weeks later when cachexia appeared in some of the animals. All mice were dissected and/or observed with GFP imaging system. The organs were collected for histological analysis of metastases. The number of metastatic nodules per organ was counted under a dissecting microscope.
Statistical analysis. All statistical analyses were performed by SPSS 13.0 for Windows (SPSS, Inc., Chicago, IL, USA). The Mann-Whitney U test was used to analyze the relationship between Tiam1 expression and clinicopathological characteristics. Survival curves were plotted by the Kaplan-Meier method and compared by the log-rank test. The significance of various variables for survival was analyzed by the Cox proportional hazards model in the multivariate analysis. Differences between groups were analyzed by a one-way analysis of variance (ANOVA) or a Student's t-test; multiple comparison of the mean was performed by variance analysis; bivariate relevant information was analyzed using correlation coefficient. $\mathrm{P}<0.05$ in all cases was considered to indicate a statistically significant result.

\section{Results}

Tiaml is overexpressed in NPC tissues. To investigate the correlation of Tiam1 expression with human NPC, we first analyzed Tiam1 protein expression in 140 paraffin-embedded, archival NPC tissues by immunohistochemical staining. As shown in Fig. 1, Tiam1 staining was mainly localized to the cytoplasm and the cytoplasmic membrane of carcinoma (Fig. 1B-D) and endothelial cells (Fig. 1E). Overexpression of Tiam1 protein was detected in 96 of 140 (68.6\%) NPC tissues, compared with 14 of 30 (46.67\%) normal nasopharynx tissues. A significant difference in overexpression was found for tumor and normal tissue samples $(\mathrm{P}=0.023)$.

Increased expression of Tiam1 correlates with progression of NPC. The association between Tiam1 expression and clinicopathological characteristics of tumors was examined. As summarized in Table I, there was no significant correlation between Tiam1 expression and gender, age, pathological classification or $\mathrm{T}$ classification $(\mathrm{P}>0.05)$. However, Tiam1 expression was positively correlated with $\mathrm{N}$ classification $(\mathrm{P}=0.004)$, distant metastasis $(\mathrm{P}=0.042)$ and clinical stage $(\mathrm{P}=0.042)$.

Tiam1 expression is inversely correlated with prognosis in NPC patients. To investigate the prognostic value of Tiam1 for NPC, we evaluated the association between Tiam1 expression and survival duration using Kaplan-Meier analysis with the log-rank test. Tiam1 expression in NPC was significantly correlated with overall survival $(\mathrm{P}=0.002)$ (Fig. 1F). The log-rank test showed that the survival time was significantly different between groups with high and low expression of Tiam1, indicating that the high level of Tiam1 correlated with shorter survival time.

To determine whether the expression of Tiam 1 is an independent prognostic factor of outcomes, multivariate survival analysis, which included gender, age, $\mathrm{T}$ classification, $\mathrm{N}$ classification, clinical stage and Tiam1 expression, was carried out. Results showed that the expression of Tiam1 protein had a significant correlation with prognosis and was an independent prognostic factor of outcomes of NPC (Table II).

Analysis of the expression of Tiaml in NPC cell lines by $R T$-PCR and immunofluorescence staining. To investigate the levels of expression and subcellular location of Tiam1 in NPC 

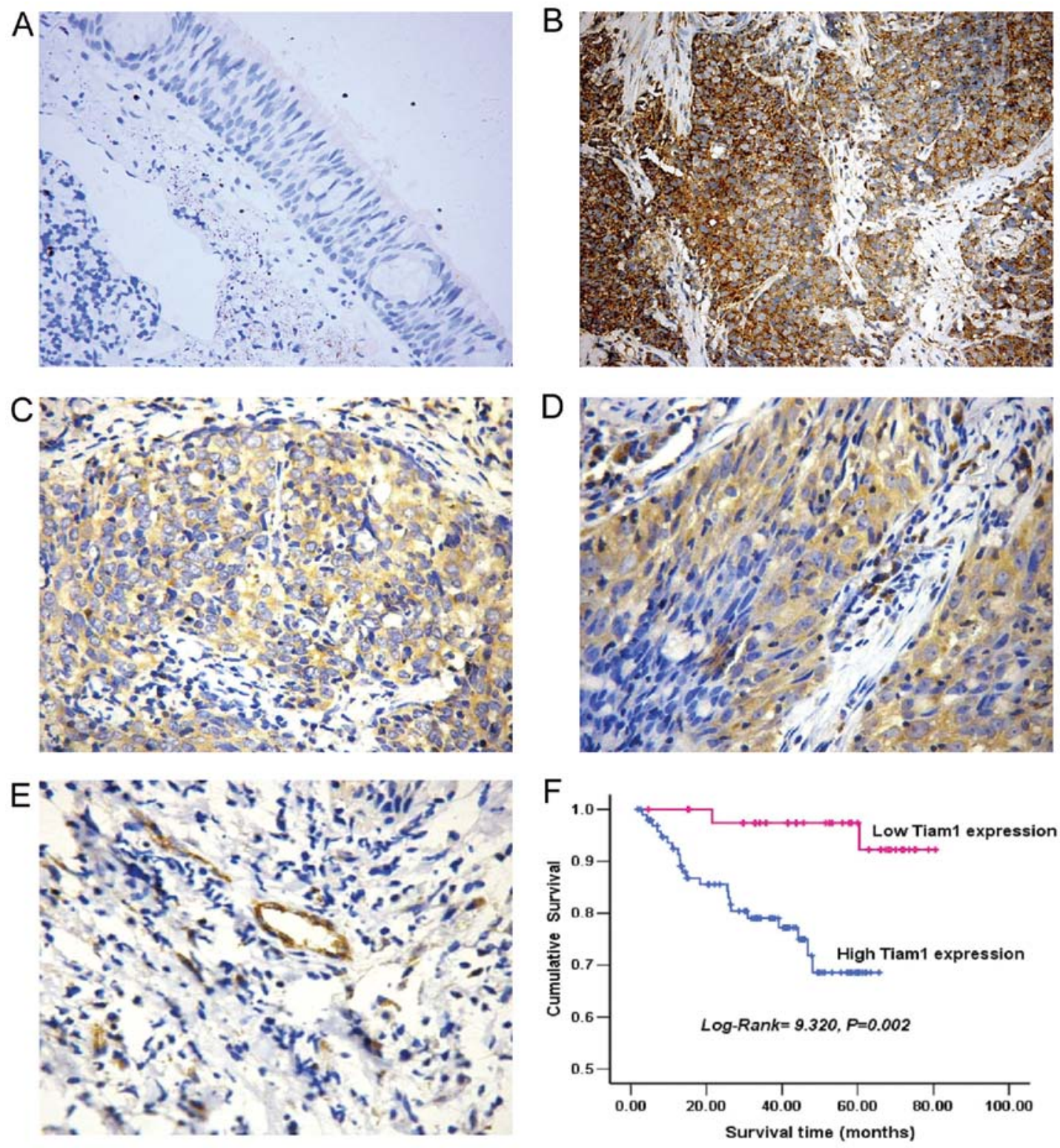

Figure 1. Expression analysis of Tiam1 protein in tissues by immunohistochemistry and Kaplan-Meier plot of overall survival duration in patients with NPC. (A) Tiam1 staining of normal nasopharyngeal epithelial tissue (original magnification, $\mathrm{x} 200$ ). (B-D) Positive expression of Tiam1 in NPC samples (original magnification, x200, x400, x400). (E) Positive control of Tiam1 in endothelial cells (original magnification, x200). (F) Kaplan-Meier survival analysis of overall survival duration in all patients according to Tiam1 expression. The log-rank test was used to calculate P-values.

cell lines, RT-PCR and immunofluorescence staining were performed. Tiam1 was highly expressed in $5-8 \mathrm{~F}$ cells, lowly expressed in 6-10B, and at a moderate level in the other 4 NPC cell lines (Fig. 2A and B). Tiam1 protein was mainly located in the perinuclear cytoplasm and cytoplasmic membrane of the NPC cells (Fig. 2C). The expression intensity of Tiam1 protein shown by immunofluorescence staining in NPC cells was consistent with the results of RT-PCR.

Alteration of Tiaml expression affects proliferation and colony formation of NPC cells in vitro. To further determine whether Tiam1 affects the biological behaviors of NPC cells, we generated two cell clones with stably overexpressed Tiam1 or with stably knocked down endogenous Tiam1. CNE2 cells were stably transfected with C1199/Tiam1 plasmids or infected with viral supernatants containing shRNA, and then were transformed into $\mathrm{CNE} 2 / \mathrm{Tiam}^{+}{ }^{+}$and $\mathrm{CNE} 2 / \mathrm{Tiam} 1^{\text {down }}$ cells, respectively.

We first examined the effect of increased Tiam1 expression on NPC cell growth in vitro. Using MTT assay, we found that parental wild-type CNE2 (WT) cells had a similar growth rate as $\mathrm{CNE} 2 /$ mock cells over a 7-day period, while starting from day 3 the growth rate of $\mathrm{CNE} 2 / \mathrm{Tiam}^{+}$cells was significantly faster than that of the former two cell lines $(\mathrm{P}<0.05)$ (Fig. 3A). Notably, plate colony formation assay showed that CNE2/Tiam1 ${ }^{+}$cells had a stronger ability to form colonies than CNE2 or mock cells (Fig. 3B). The consistent results also appeared in the soft agar assay. The colony formation rates of CNE2/Tiam $1^{+}, \mathrm{CNE} 2 /$ mock and WT cells were $86.44 \pm 1.98,59.22 \pm 2.40$ and $57.94 \pm 3.50 \%$, respectively. CNE2/Tiam $1^{+}$cells had a higher efficiency to form colonies than the other two groups, suggesting that overexpression of 

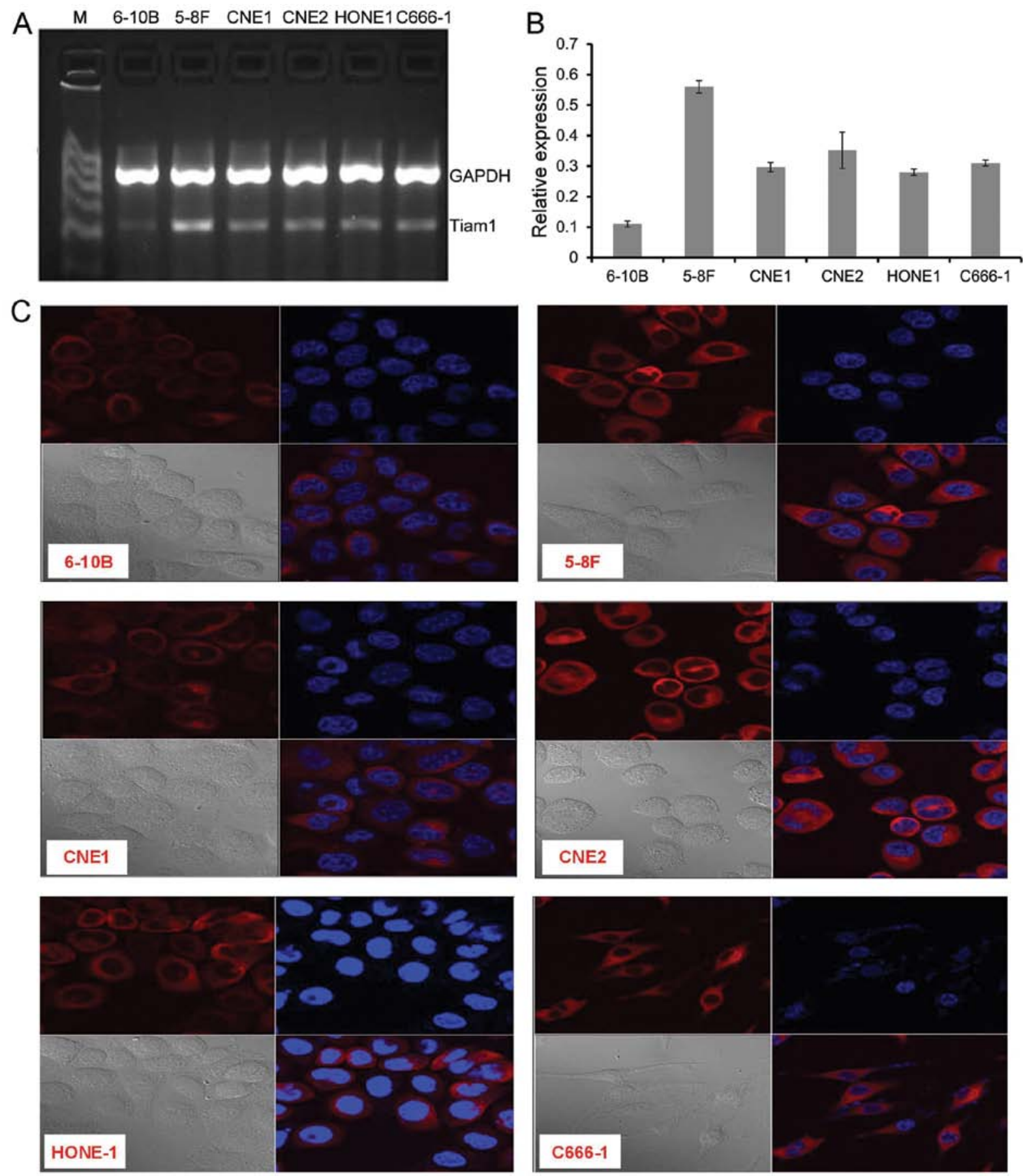

Figure 2. Expression analysis of Tiam1 in NPC cell lines by RT-PCR and immunofluorescence staining. (A) RT-PCR analysis of Tiam1 expression in 6 NPC cell lines. (B) The expression levels of Tiam1 gene relative to GAPDH were calculated. Error bars indicate SD, n-3 experiments. (C) Immunofluorescence staining of Tiam1 expression in 6 NPC cell lines stained with anti-Tiam1 (red) and 4,6-diamidino-2-phenylindole (DAPI, to stain DNA; blue).

Tiam1 promoted the anchorage-independent growth ability of CNE2 cells (Fig. 3C). In contrast, knockdown of endogenous Tiam1 markedly reduced the colony formation ratio of CNE2 cells. The colony formation ratio of CNE2/Tiam ${ }^{\text {down }}$ cells was reduced by $\sim 80 \%$, as compared with that of WT and shRNAcontrol cells $(\mathrm{P}<0.001)$ (Fig. 3D). The above results indicate that increased Tiam1 expression promotes the proliferation and colony formation of NPC cells.

Alteration of Tiaml expression affects the adhesion, invasion and migration of NPC cells in vitro. We next focused on determining the effects of Tiam1 on cell adhesion, invasion and migration activity in vitro. Effects of Tiam1 overexpression or knockdown on adhesion and invasion capacities of NPC cells were assayed using an adhesion assay and ECMatrix invasion assay. The adhesion assay revealed that the adhesive ability to fibronectin of $\mathrm{CNE} 2 / \mathrm{Tiam}^{+}$cells increased by nearly $50 \%$ as compared with the WT and mock cells (Fig. 4A). ECMatrix invasion assay revealed that the number of CNE2/Tiam $1^{+}$, mock and WT cells that penetrated through the matrix was $104.00 \pm 5.12,53.44 \pm 4.56$ and $51.33 \pm 5.22$, respectively. Compared with the mock and WT cells, CNE2/ 
Table II. Summary of overall survival analyses by univariate and multivariate COX regression analysis.

\begin{tabular}{|c|c|c|c|c|c|c|}
\hline \multirow[b]{2}{*}{ Parameters } & \multicolumn{3}{|c|}{ Univariate analysis } & \multicolumn{3}{|c|}{ Multivariate analysis } \\
\hline & P-value & HR & $95 \% \mathrm{CI}$ & P-value & HR & $95 \% \mathrm{CI}$ \\
\hline Age (years) & & & & & & \\
\hline$>45$ vs. $\leq 45$ & 0.463 & 1.364 & $0.596-3.124$ & & & \\
\hline Gender & & & & & & \\
\hline Male vs. female & 0.104 & 0.367 & $0.109-1.230$ & & & \\
\hline $\begin{array}{l}\text { Histologic classification } \\
\text { Type II vs. type III }\end{array}$ & 0.185 & 0.441 & $0.131-1.479$ & & & \\
\hline $\begin{array}{l}\text { T classification } \\
T_{1}-T_{2} \text { vs. } T_{3}-T_{4}\end{array}$ & 0.025 & 2.582 & $1.129-5.903$ & 0.666 & 0.809 & $0.309-2.117$ \\
\hline $\begin{array}{l}\mathrm{N} \text { classification } \\
\mathrm{N}_{0} \text { vs. } \mathrm{N}_{1}-\mathrm{N}_{3}\end{array}$ & 0.094 & 2.016 & $0.888-4.577$ & & & \\
\hline $\begin{array}{l}\text { M classification } \\
\mathrm{M}_{0} \text { vs. } \mathrm{M}_{1}\end{array}$ & 0.000 & 5.728 & $2.250-14.585$ & 0.013 & 3.395 & $1.295-8.897$ \\
\hline $\begin{array}{l}\text { Clinical stage } \\
\text { I-II vs. III-IV }\end{array}$ & 0.003 & 6.391 & $1.906-21.433$ & 0.022 & 5.149 & $1269-20.890$ \\
\hline $\begin{array}{l}\text { Tiam1 expression } \\
\text { Negative vs. positive }\end{array}$ & 0.008 & 7.417 & $1.689-32.564$ & 0.031 & 5.029 & $1.158-21.845$ \\
\hline
\end{tabular}
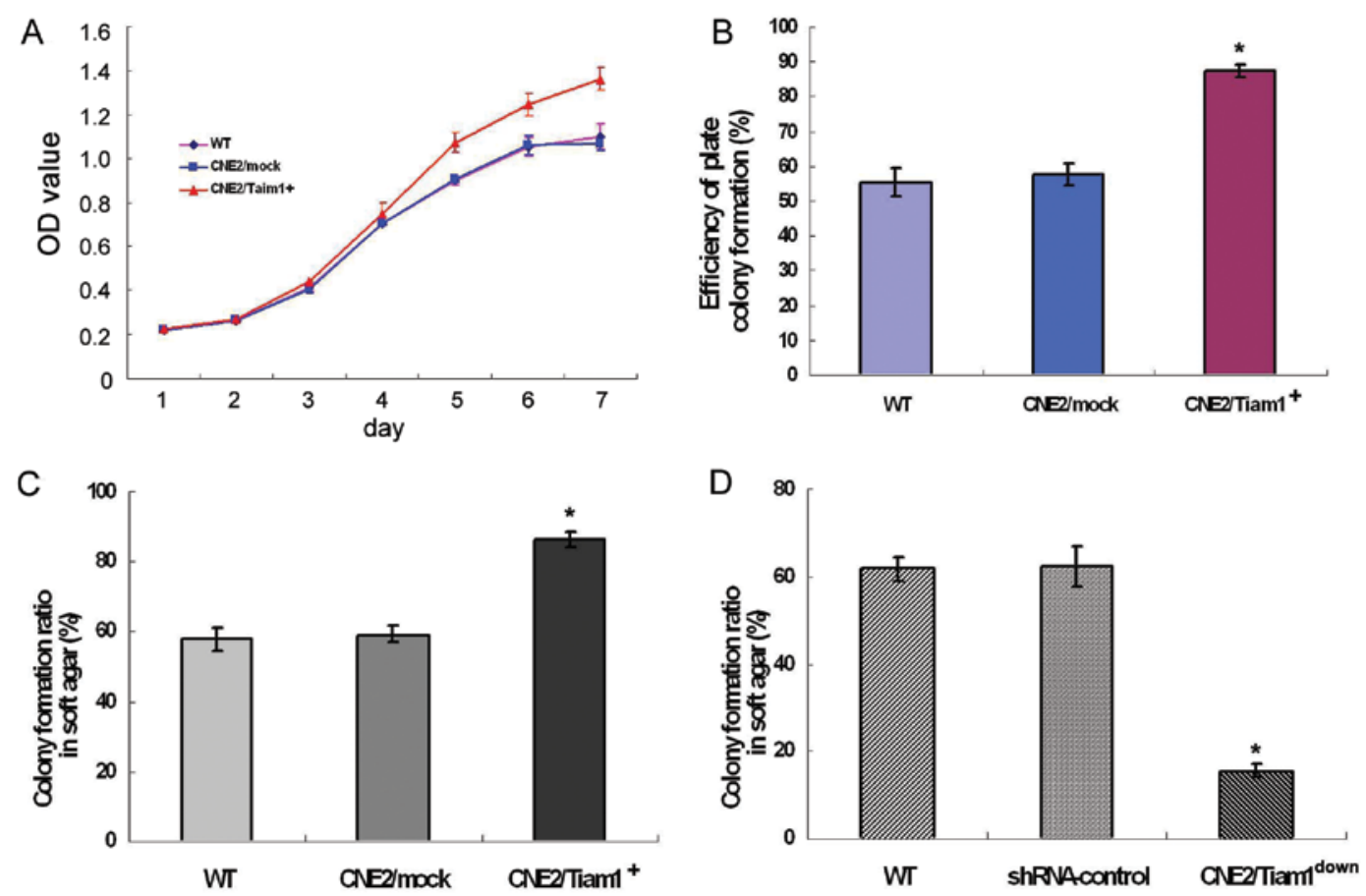

Figure 3. Alteration of Tiam1 expression affects proliferation and colony formation of NPC cells in vitro. (A) The cell growth of parental WT, CNE2/mock and CNE2/Tiam $1^{+}$cells was examined by MTT assay throughout a 7-day period. (B) The ability of the WT, CNE2/mock and CNE2/Tiam $1^{+}$cells to form colonies was determined by a plate colony formation assay. (C) Comparison of the single-cell reproductive capability and colony formation ratio in soft agar of WT, CNE2/mock and CNE2/Tiam1 ${ }^{+}$cells. (D) Comparison of the single-cell reproductive capability and colony formation ratio in soft agar of WT, shRNA-control and CNE2/Tiam1 1own cells. WT, wild-type CNE2 cells. CNE2/mock, CNE2 cells that were transfected stably by pcDNA3.1 vector. CNE2/Tiam1 ${ }^{+}, \mathrm{CNE} 2$ cells that exogenously overexpressed Tiam1 by stable transfection with the Tiam1/C1199HA vector. shRNA-control, CNE2 cells that were infected with the viral supernatants containing negative control shRNA. CNE2/Tiam1 ${ }^{\text {down }}$, CNE2 cells that had disrupted endogenous Tiam1 by knockdown technique ("P $<0.05$; ANOVA test with LSD post hoc test).

$\mathrm{Tiam}^{+}$cells exhibited markedly greater penetration potency $(\mathrm{P}<0.05)$ (Fig. 4B). The opposite results were observed in the experimental groups with disruption of endogenous Tiam1 expression. The Tiam1-knockdown cells exhibited only a 

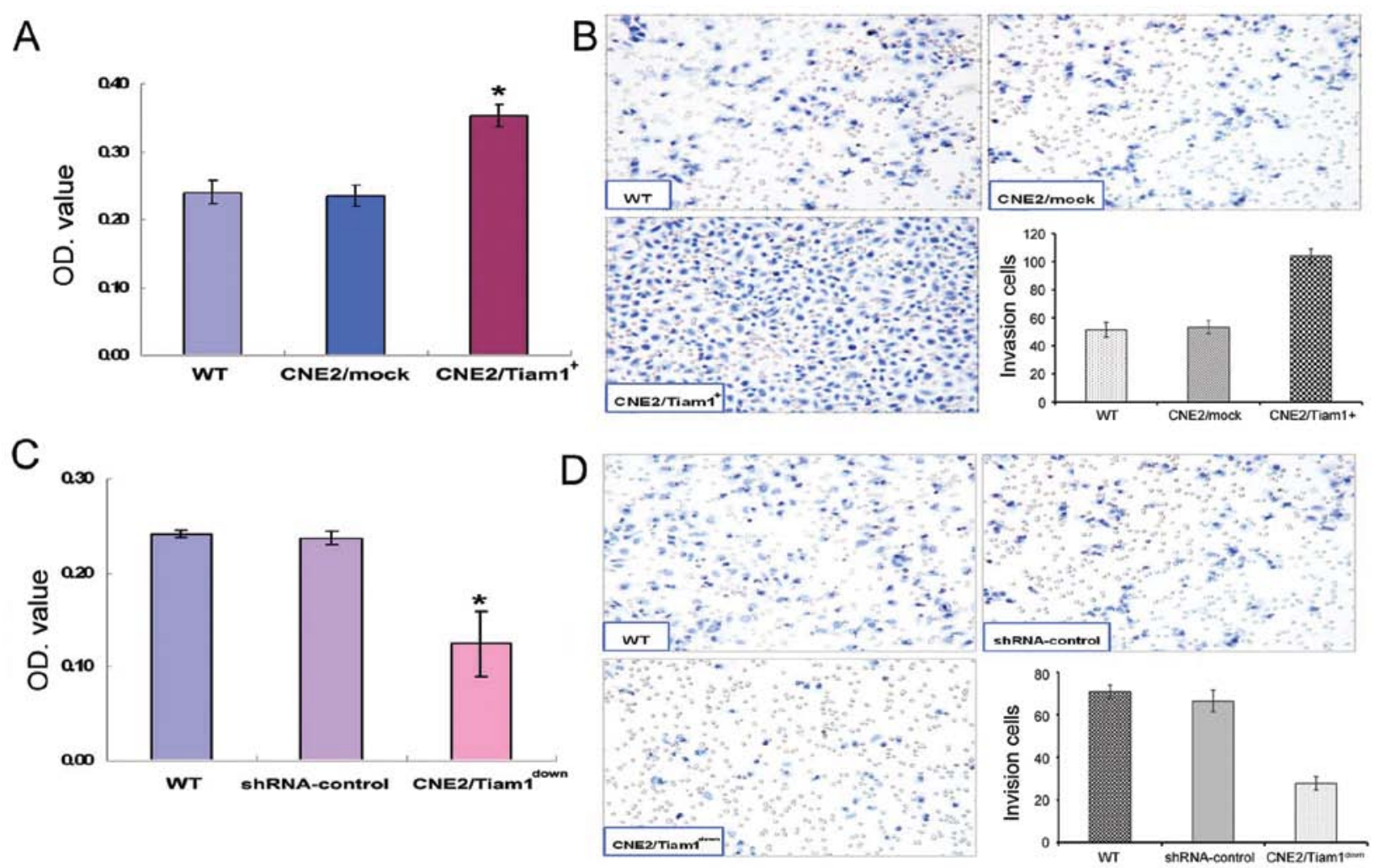

$\mathrm{E}$ $4 \mathrm{~h}$

$18 \mathrm{~h}$

$30 \mathrm{~h}$
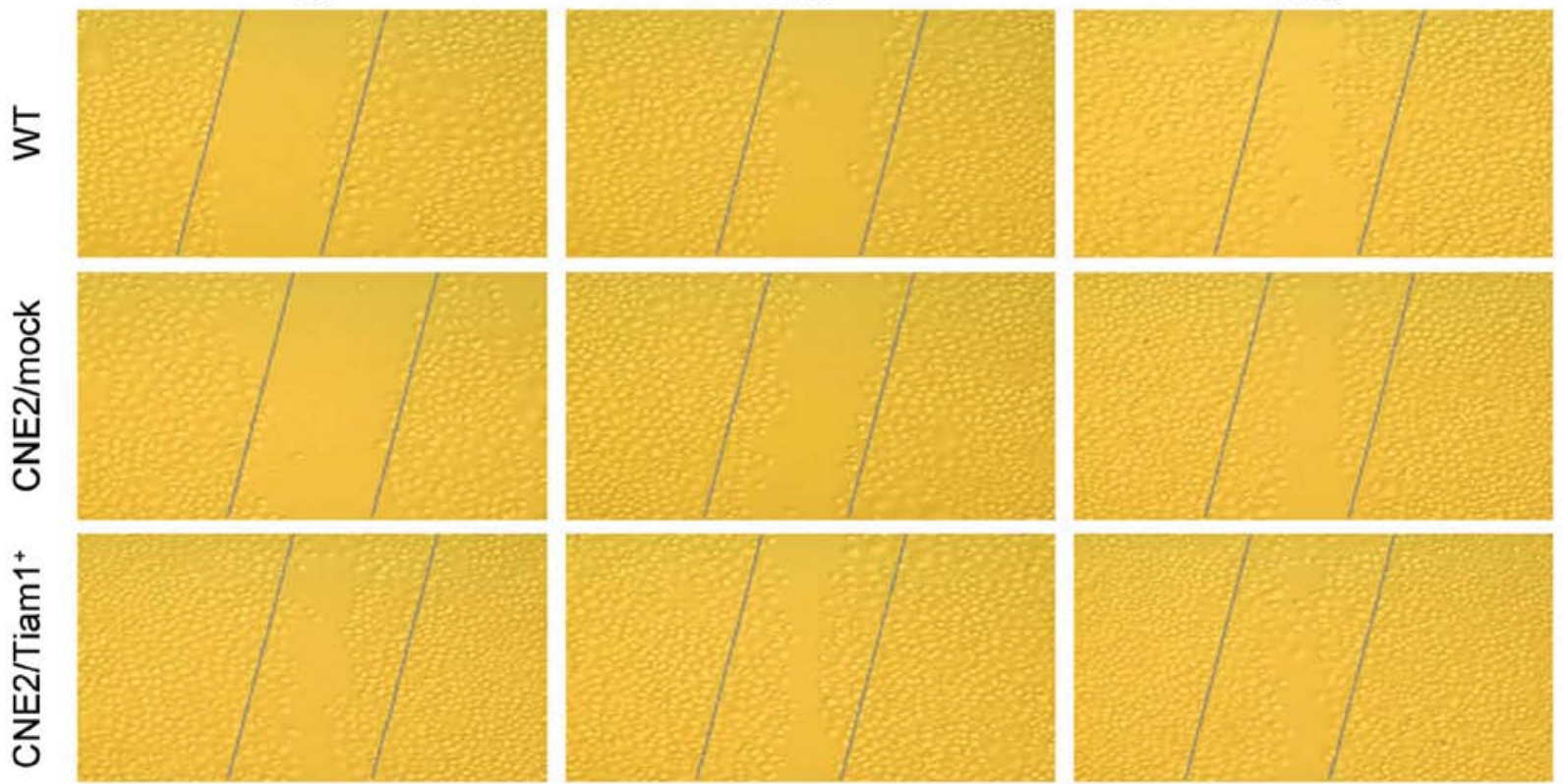

Figure 4. Alteration of Tiam1 expression affects the adhesion, invasion and migration of CNE2 cells in vitro. (A and C) Cell adhesion and (B and D) invasive capabilities of CNE2/Tiam1 ${ }^{+}$, CNE2/Tiam1 ${ }^{\text {down }}$ and the corresponding control cells were examined using an adhesion assay and Transwell assay. (E) Effect of increased Tiam 1 expression on the migratory ability of CNE2 cells was determined using a scratch-wound healing assay. After 4 , 18 and $30 \mathrm{~h}$ of wounding, the number of cells that migrated into the scratched area from the wound edge was recorded and analyzed (*P<0.05; ANOVA test with LSD post hoc test).

nearly 0.5 -fold adhesion ability when compared with the WT or shRNA-control cells $(\mathrm{P}<0.05)$ (Fig. 4C). ECMatrix invasion assay demonstrated that Tiam 1 shRNA cells displayed a significant decrease in invasive ablity, as compared with that of the WT and shRNA-control cells $(\mathrm{P}<0.05)$ (Fig. 4D). The results imply that the abilities of adhesion and invasion of NPC cells are correlated with the alteration of Tiam1 expression.
The upregulation or downregulation of Tiam1 expression enhances or attenuates the adhesion and invasion of NPC cells.

The effect of Tiam 1 overexpression on the migratory ability of CNE2 cells was determined using a scratch-wound healing assay (Fig. 4E). After 4, 18 and 30 h of wounding, the number of migrated cells in the wound space was counted in 3 randomly selected fields. The experiments were repeated 

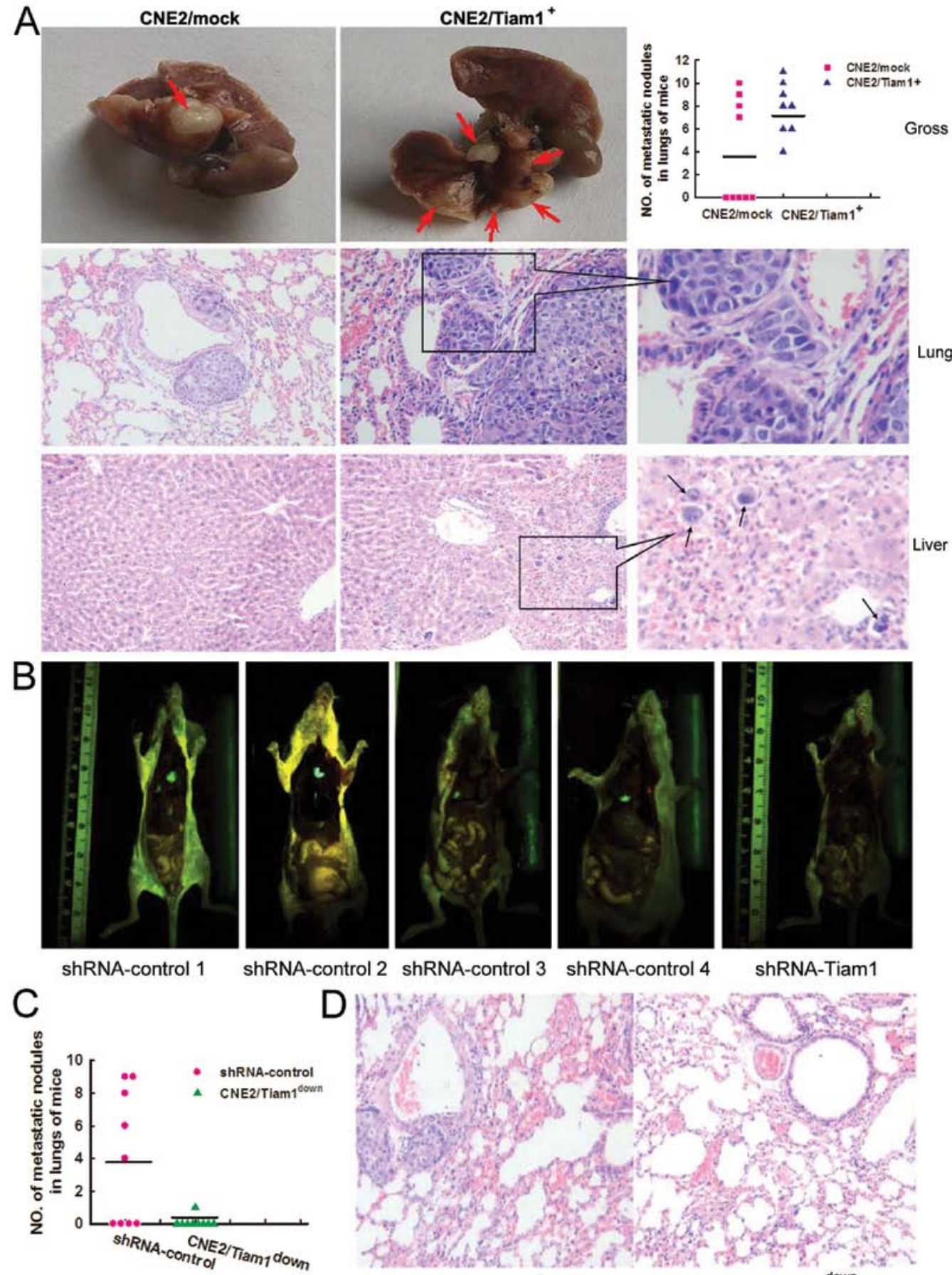

shRNA-control

shRNA-Tiam1

ShRNA-control 4
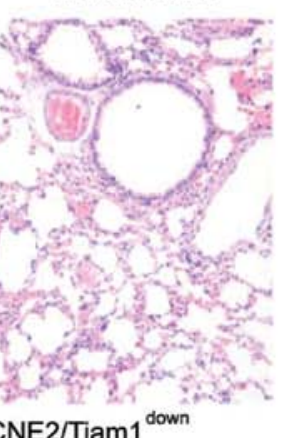

Figure 5. Alteration of Tiam1 expression affects CNE2 metastasis in vivo. (A) Marked numbers of metastatic nodules in the lung and liver of mice were confirmed by macrography (red arrows) and histopathological staning. Left panel, experiment group injected with CNE2/mock cells. Middle panel, experimental group injected with CNE2/Tiam1 ${ }^{+}$cells. (B) Whole-body fluorescence images of metastatic nodules of shRNA-control cells and CNE2/Tiam1 ${ }^{\text {down }}$ cells were obtained following injection into nude mice. (C) Numbers of metastatic nodules in the lungs of mice following injection of shRNA-control CNE2 and CNE2/ Tiamldown cells, respectively. (D) The gross findings were confirmed by histological observation. Metastatic nodules were observed in the lungs of mice following injection of shRNA-control cells by histopathological staning.

in triplicates. The number of WT, CNE2/mock and CNE2/ Tiam $1^{+}$cells that migrated to the wound area from the edge 4 , 18 and $30 \mathrm{~h}$ after the scratching was as follows: $4 \mathrm{~h}, 27.67 \pm 6.03 /$

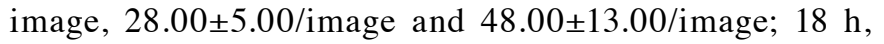
$47.00 \pm 4.58 /$ image, $45.67 \pm 5.67 /$ image and $96.33 \pm 13.50 /$ image; $30 \mathrm{~h}, 78.00 \pm 4.58 / \mathrm{image}, 86.33 \pm 10.07 / \mathrm{image}$ and $141.00 \pm 15.14 /$ image, respectively. The number of migratory cells was significantly increased in the $\mathrm{CNE} 2 / \mathrm{Tiam}^{+}$group as compared with that of the WT and mock groups $(\mathrm{P}<0.05)$, demonstrating that the migratory ability of NPC cells was positively correlated with increased Tiam1 expression.

Alteration of Tiam1 expression affects NPC metastasis in vivo. In addition to examining the biological functions of Tiam1 in vitro, we also assessed the in vivo function of Tiam1 by establishing an animal metastasis model by intravenously 
injecting CNE2/Tiam1 $1^{+}$, CNE2/Tiam1 ${ }^{\text {down }}$ and corresponding control cells into nude mice, respectively. In the mice injected with the CNE2/Tiam $1^{+}$cells, one died due to abscesses in multiple organs 15 days after injection. Two months later when cachexia occurred in the CNE2/Tiam1 ${ }^{+}$group, all of the remaining mice were sacrificed. Autopsy revealed that nude mice in the CNE2/Tiam $1^{+}$group had many more metastatic nodules in lungs than those in the CNE2/mock group, indicating extravasation and tumor growth in the lungs (Fig. 5A). In the CNE2 mock group, 4 of 9 (44\%) mice developed metastasis in the lungs, while all $8(100 \%)$ mice in the CNE2/Tiam $1^{+}$group displayed marked numbers of metastatic nodules in the lungs (Fig. 5A). The results were confirmed by histopathological staining (Fig. 5A). Furthermore, scattered NPC cells were found in the liver in one of the CNE2/Tiam $1^{+}$ group mice by microscopy (Fig. 5A). No detectable tumors were found in other organs in mice in either the CNE2/Tiam $1^{+}$ or CNE2/mock group. In the groups of mice injected with CNE2/Tiam1 ${ }^{\text {down }}$ or shRNA-control CNE2 cells, 5 of 9 mice in the shRNA-control group developed collectively 36 metastatic nodules in the lungs, indicating extravasation of tumor growth. In contrast, the 9 mice injected with Tiam1 shRNA cells developed only one metastatic nodule in the lungs as determined using a GFP imaging system (Fig. 5B and C). No metastatic nodules were found in the other organs. The gross findings were further confirmed by histological observation (Fig. 5D). These data showed that Tiam1 promotes the metastasis of NPC cells in vivo, which was validated both by overexpression and knockdown experiments.

\section{Discussion}

NPC is a rare malignancy in most regions of the world, while it is highly prevalent in southern Asia where the disease occurs at an approximately 100-fold higher incidence compared with other populations not at risk. NPC is one of the most confusing, commonly misdiagnosed and poorly understood disease. Significant achievements in the basic sciences have led to a greater knowledge of the underlying molecular genetics of NPC, which holds promise in attempts to tailor patient prognostication and for future treatment strategies. An enhanced ability to predict patient survival will allow for better selection of patients most likely to benefit from systemic therapies and for more accurate comparison of clinical trials. Tiam1, an important member of the Rho GTPase family, was identified to play an important role during the invasion and metastasis of many types of cancer cells (7-11,19-21). Engers et al (22) found that significantly increased Tiam1 expression levels were observed in both prostate cancer and almost all preneoplastic high-grade prostatic intraepithelium neoplasia lesions, indicating that increased Tiam1 expression may occur early in prostate carcinoma development. Huang et al (21) and Liu et al (23) clarified the possible role of the Tiam1 gene in the proliferation, invasion and metastasis of colorectal cancer (CRC) and hepatocellular carcinoma (HCC), respectively, and established Tiam1 as a new target for early metastatic diagnostic markers. Mo et al (19) and Zhang et al (20 both reported that that overexpression of Tiam1 was correlated with invasion and metastasis of NPC; however, little is known about the potential prognostic value and molecular mechanisms of Tiam1 in NPC.
In the present study, we assessed Tiam1 expression in NPC using an immunochemical staining approach and explored the clinical prognostic value of Tiam1 by using complete longterm follow-up data of a large cohort of patients with NPC. We found that $\sim 68.6 \%$ (96 of 140 cases) of NPC tissues overexpressed Tiam1 protein. The positive rate of Tiam1 protein was higher in NPC than that in normal nasopharyngeal tissues. We further confirmed that Tiam1 was overexpressed in NPC tissues. Furthermore, we also found that Tiam1 overexpression was significantly associated with $\mathrm{T}, \mathrm{M}$ classification and clinical stage of NPC patients. In addition, the expression of Tiam1 was detected in all of the 6 NPC cell lines. As indicated by RT-PCR and immunofluorescence staining, the highly metastatic cell line, $5-8 \mathrm{~F}$, exhibited the highest staining of Tiam1, while the non-metastatic 6-10B cell line showed the lowest expression of Tiam1. Our finding, in agreement with many other observation of overexpression of Tiam1 in a variety of cancer tissues, including NPC, confirmed the close association between Tiam1 expression and progression and metastasis of tumors.

Our analysis further indicated that Tiam1 protein expression was inversely correlated with overall survival of NPC patients. The higher the expression of Tiam1, the shorter was the survival time for patients with NPC. By univariate analysis of Cox proportional-hazards model, Tiam1 expression, $\mathrm{T}$ classification, $\mathrm{M}$ classification and clinical stage were found to be associated with an increased risk of death from NPC. In the multivariate analyses, a high expression of Tiam1 protein was a significant predictor of poor prognosis for NPC patients. These results suggest the clinical significance of Tiam1 as a biomarker for NPC prognosis.

The expression of Tiam1 has been widely studied in various human cancers. Most previous reports indicate that upregulation of Tiam1 is associated with metastasis and serves as an unfavorable prognostic factor in cancers $(8,11,22-26)$. However, contradicting results were also observed in various studies. Engers et al (27) found that two invasive cell lines either failed to express Tiam1 or exhibited very low expression levels of Tiam1, but expression of Tiam1 was markedly higher in the intermediate or low invasive potential cell lines in renal cell carcinona. Uhlenbrock et al (12) found that Tiam1 inhibited migration and invasion of metastatic melanoma via a novel adhesive mechanism. In gastric carcinoma, increased expression of Tiam1 was found in half of the examined gastric carcinomas, tending to be associated with favorable prognosis (28). All of these data suggest a complex role of Tiam1 in different types of human cancers. Thus, it is important to elucidate the precise roles and molecular mechanisms of Tiam1 in NPC tumorigenesis and development. To understand the biological function of Tiam1, we employed two different approaches to alter the expression level of endogenous Tiam1 in NPC cells, that is, by the exogenous overexpression and the knockdown of expression of Tiam1. CNE2 cells exhibited a moderate expression level of endogenous Tiam 1 among all the 6 NPC cell lines examined, and thus, represent an ideal model for the present study.

In the present study, we determined that overexpression of Tiam1 enhanced the abilities of proliferation and colony formation of CNE2 cells. On the other hand, the present study demonstrated that an efficient knockdown of the Tiam1 gene 
strongly inhibited in vitro cell growth and colony formation efficiency. The soft agar colony formation experiment is well known for determining non-anchored capacity for growth of a single cell, reflecting the growth characteristics of tumor cells in vivo. The present study showed that the colony formation efficiency was enhanced in Tiam1-overexpressing NPC cells while reduced in Tiam1-silencing NPC cells. This indicates that Tiam1 may be a positive regulator of tumor growth and a significant modulator of tumor development in NPC.

Since Tiam1 is known as a metastasis-associated gene in other types of cancer, the effects of Tiam1 overexpression/knockdown on the metastatic potential of NPC cells were also assessed in the present study. The migratory and invasive ability, an important aspect of epithelial cells, depends predominantly on cell migration and cell-substrate adhesion. The cellular ability of attaching to extracellular matrix (ECM) components contributes to the invasion and metastasis of tumor cells, for heterogeneous adhesion can be mediated by interactions between tumor cells and the host or matrix adhesion molecules. We observed that knockdown of Tiam1 expression in NPC cells reduced their ability to adhere to fibronectin in our adhesion assay and to invade ECMatrix-coated membranes in our invasion chamber assay. This is opposite to our observation that overexpression of Tiam1 in NPC cells improves their abilities to adhere to fibronectin and to invade ECMatrix-coated membrane. Our experiments also demonstrated that overexpression of Tiam1 enhanced the ability of cell migration by scratch wound assay in confluent monolayer NPC cells, as was observed by Minard et al (29) in CRC. These results suggest that Tiam1 is required for the invasive phenotypes of NPC cells in vitro. To prove that Tiam1 plays an important role in promoting NPC cell metastasis in vivo as it did in vitro, we also constructed experimental metastasis models. The experimental results clearly demonstrated that increased expression of Tiam 1 enhanced the ability of NPC cells to metastasize to and grow in the lungs and liver of mice, while reduction of Tiam1 expression abrogated such ability of NPC cells. The positive relation of Tiam1 expression to the metastatic potential of NPC cells provides another piece of evidence for the crucial role of Tiam1 in NPC progression. These results strongly suggest that Tiam 1 overexpression is associated with the malignant phenotype of NPC.

In conclusion, this is the first relative comprehensive study showing the functional mechanisms of Tiam1 in NPC, highlighting the clinical significance of Tiam1 in NPC. The present study revealed that the level of expression of Tiam1 was highly increased in NPC tissues, and Tiam1 overexpression was inversely correlated with survival and directly correlated with malignant status of patients with NPC. Tiam1 could be used as a valuable molecular marker for NPC and an indicator for tumor progression and invasion. Moreover, we identified the roles of Tiam1 in promoting cell proliferation, adhesion, invasion and migration in vitro and in vivo. These observations could provide new insight into understanding the molecular mechanisms involved in NPC progression and prognosis, and may result in the development of novel therapeutic strategies for NPC. However, additional research is needed to investigate the mechanisms and pathways of NPC pathogenesis mediated by Tiam1.

\section{Acknowledgements}

The present study was supported by the National Natural Science Fund of China (grant nos. 30800414, 30801380, 30770977, 30670967, 30670968, 30500242, 81071735 and 81000953) and the Nature Science Fund of Guangdong Province of China (nos. 5200512, 2010B031500012 and 10451051501004710).

\section{References}

1. Her C: Nasopharyngeal cancer and the Southeast Asian patient. Am Fam Physician 63: 1776-1782, 2001

2. Lo KW and Huang DP: Genetic and epigenetic changes in nasopharyngeal carcinoma. Semin Cancer Biol 12: 451-462, 2002.

3. Cvitkovic E, Bachouchi M, Boussen $\mathrm{H}$, et al: Leukemoid reaction, bone marrow invasion, fever of unknown origin, and metastatic pattern in the natural history of advanced undifferentiated carcinoma of nasopharyngeal type: a review of 255 consecutive cases. J Clin Oncol 11: 2434-2442, 1993.

4. Chan AS, To KF, Lo KW, et al: High frequency of chromosome $3 p$ deletion in histologically normal nasopharyngeal epithelia from southern Chinese. Cancer Res 60: 5365-5370, 2000.

5. Loong HH, Ma BB and Chan AT: Update on the management and therapeutic monitoring of advanced nasopharyngeal cancer. Hematol Oncol Clin North Am 22: 1267-1278, 2008.

6. Habets GG, Scholtes EH, Zuydgeest D, et al: Identification of an invasion-inducing gene, Tiam-1, that encodes a protein with homology to GDP-GTP exchangers for Rho-like proteins. Cell 77: 537-549, 1994.

7. Jeng YM, Chang CC, Hu FC, et al: RNA-binding protein insulin-like growth factor II mRNA-binding protein 3 expression promotes tumor invasion and predicts early recurrence and poor prognosis in hepatocellular carcinoma. Hepatology 48: 1118-1127, 2008.

8. Hou M, Tan L, Wang X and Zhu YS: Antisense Tiam1 downregulates the invasiveness of $95 \mathrm{D}$ cells in vitro. Acta Biochim Biophys Sin (Shanghai) 36: 537-540, 2004.

9. Minard ME, Kim LS, Price JE and Gallick GE: The role of the guanine nucleotide exchange factor Tiam 1 in cellular migration, invasion, adhesion and tumor progression. Breast Cancer Res Treat 84: 21-32, 2004.

10. Minard ME, Herynk MH, Collard JG and Gallick GE: The guanine nucleotide exchange factor Tiam1 increases colon carcinoma growth at metastatic sites in an orthotopic nude mouse model. Oncogene 24: 2568-2573, 2005.

11. Ding Y, Chen B, Wang S, et al: Overexpression of Tiam1 in hepatocellular carcinomas predicts poor prognosis of $\mathrm{HCC}$ patients. Int J Cancer 124: 653-658, 2009.

12. Uhlenbrock K, Eberth A, Herbrand U, et al: The RacGEF Tiam1 inhibits migration and invasion of metastatic melanoma via a novel adhesive mechanism. J Cell Sci 117: 4863-4871, 2004.

13. Song LB, Yan J, Jian SW, et al: Molecular mechanisms of tumorgenesis and metastasis in nasopharyngeal carcinoma cell sublines. Ai Zheng 21: 158-162, 2002 (In Chinese).

14. Masunaga R, Kohno H, Dhar DK, et al: Cyclooxygenase-2 expression correlates with tumor neovascularization and prognosis in human colorectal carcinoma patients. Clin Cancer Res 6: 4064-4068, 2000.

15. Soumaoro LT, Uetake H, Higuchi T, Takagi Y, Enomoto M and Sugihara K: Cyclooxygenase-2 expression: a significant prognostic indicator for patients with colorectal cancer. Clin Cancer Res 10: 8465-8471, 2004.

16. Wang S, Zhou J, Wang XY, et al: Down-regulated expression of SATB2 is associated with metastasis and poor prognosis in colorectal cancer. J Pathol 219: 114-122, 2009.

17. Rodriguez M, Aladowicz E, Lanfrancone L and Goding CR: Tbx3 represses E-cadherin expression and enhances melanoma invasiveness. Cancer Res 68: 7872-7881, 2008.

18. Herve MA, Buteau-Lozano H, Vassy R, et al: Overexpression of vascular endothelial growth factor 189 in breast cancer cells leads to delayed tumor uptake with dilated intratumoral vessels. Am J Pathol 172: 167-178, 2008.

19. Mo L, Wang H, Huang G, Zhao H and Kuang G: Correlation between expression of the Tiam1 gene and the invasion and metastasis in nasopharyngeal carcinoma. Lin Chuang Er Bi Yan Hou Ke Za Zhi 19: 785-787, 2005 (In Chinese). 
20. Zhang XM, Ding Y, Chen JZ, et al: Overexpression of Tiam1 gene and its relationship with invasive and metastatic ability of nasopharyngeal carcinoma. Zhonghua Bing Li Xue Za Zhi 38: 268-272, 2009 (In Chinese).

21. Huang J, Ye X, Guan J, et al: Tiam1 is associated with hepatocellular carcinoma metastasis. Int J Cancer 132: 90-100, 2013.

22. Engers R, Mueller M, Walter A, Collard JG, Willers R and Gabbert HE: Prognostic relevance of Tiam1 protein expression in prostate carcinomas. Br J Cancer 95: 1081-1086, 2006.

23. Liu L, Zhang Q, Zhang Y, Wang S and Ding Y: Lentivirusmediated silencing of Tiam1 gene influences multiple functions of a human colorectal cancer cell line. Neoplasia 8: 917-924, 2006.

24. Mehta SA, Christopherson KW, Bhat-Nakshatri P, et al: Negative regulation of chemokine receptor CXCR4 by tumor suppressor p53 in breast cancer cells: implications of p53 mutation or isoform expression on breast cancer cell invasion. Oncogene 26: $3329-3337,2007$
25. Zhao L, Liu Y, Sun X, He M and Ding Y: Overexpression of T lymphoma invasion and metastasis 1 predict renal cell carcinoma metastasis and overall patient survival. J Cancer Res Clin Oncol 137: 393-398, 2011.

26. Adams HC III, Chen R, Liu Z and Whitehead IP: Regulation of breast cancer cell motility by T-cell lymphoma invasion and metastasis-inducing protein. Breast Cancer Res 12: R69, 2010.

27. Engers R, Zwaka TP, Gohr L, Weber A, Gerharz CD and Gabbert HE: Tiam1 mutations in human renal-cell carcinomas. Int J Cancer 88: 369-376, 2000.

28. Walch A, Seidl S, Hermannstadter C, et al: Combined analysis of Rac1, IQGAP1, Tiam1 and E-cadherin expression in gastric cancer. Mod Pathol 21: 544-552, 2008.

29. Minard ME, Ellis LM and Gallick GE: Tiam1 regulates cell adhesion, migration and apoptosis in colon tumor cells. Clin Exp Metastasis 23: 301-313, 2006 . 